\title{
DETERMINANTS OF MODERN SCIENTIFIC DISCOURSE IN THE CONTEXT OF "SOCIALIZATION" OF KNOWLEDGE
}

\author{
Tatyana P. Tretyakova \\ St. Petersburg State University, Saint Petersburg, Russia
}

\begin{abstract}
The aim of the article is to disclose some general trends in scientific discourse studies that have emerged by the second decade of the $21^{\text {st }}$ century, to characterize scientific discourse as a means of promoting knowledge, and to consider its contribution to public communication. The notion of scientific discourse incorporates both conventional (scientific articles, reviews, theses, dissertation abstracts) and modern forms of discourse (online academic conferences, symposia, colloquiums, seminars). The research is viewed in the article as a result of scientists' social activity targeted at new knowledge discovery and broadcasting, whilst the scientific text is defined in terms of verbal representation of the research results in a par with new knowledge transmission. Two types of determinants are introduced to regulate the model of scientific text composition - internal and external. The internal determinant is represented by discursive structures, which promote knowledge in scientific text as the element of scientific communication, while the external dominant is represented by extralinguistic factors, which provide the promotion of scientific discourse outside the professional community (socializing). Both kinds of determinants provide the interpretation of the scientific discourse as open systems. An assumption is made on the increasing role of external determinant in discursive extension of modern scientific text by means of the Internet; the addressee factor wider representation and scientific knowledge inclusion into cross-cultural communication.

Key words: scientific discourse, adaptive system, determinant, modeling, knowledge formation procedure, argumentation.
\end{abstract}

Citation. Tretyakova T.P. Determinants of Modern Scientific Discourse in the Context of "Socialization" of Knowledge. Vestnik Volgogradskogo gosudarstvennogo universiteta. Seriya 2. Yazykoznanie [Science Journal of Volgograd State University. Linguistics], 2021, vol. 20, no. 5, pp. 97-107. (in Russian). DOI: https://doi.org/ 10.15688/jvolsu2.2021.5.8

\section{ДЕТЕРМИНАНТЫ СОВРЕМЕННОГО НАУЧНОГО ДИСКУРСА В КОНТЕКСТЕ «СОЦИАЛИЗАЦИИ» ЗНАНИЯ}

\author{
Татьяна Петровна Третьякова \\ Санкт-Петербургский государственный университет, г. Санкт-Петербург, Россия
}

\begin{abstract}
Аннотация. В статье определены общие тенденции лингвистического изучения научного дискурса, сложившиеся ко второму десятилетию XXI в., и охарактеризован дискурс науки с точки зрения производства _ и представления знания и его включенности в широкую коммуникацию. Понятие «научный дискурс» объединяет как традиционные (научные тексты, существующие в виде публикаций: статьи, рецензии, отзывы о диссертациях и авторефератах диссертаций и пр.), так и новые (конференции, симпозиумы, коллоквиумы, семинары и пр. в онлайн-формате) формы реализации дискурса. В статье научное исследование рассматривается как предмет социальной деятельности ученых, направленной на создание и распространение нового знания, а научный текст - как вербальное представление результатов данной деятельности и средство распространения нового знания. Описан процесс построения научного текста в рамках действия двух типов детерминант - внутренней и внешней. Внутренняя детерминанта представлена дискурсивными конструкциями, продвигающими знание в научном тексте как элементе научной коммуникации, внешняя детерминанта
\end{abstract}


представлена экстралингвистическими факторами, обеспечивающими распространение научного дискурса за пределы профессионального сообщества (социализацию). Показано, что оба вида детерминант обусловливают интерпретацию научного дискурса как открытой системы. Высказано предположение о возрастании роли внешней детерминанты в дискурсивном развертывании научного текста, проявляющейся в смене способов представления современного научного знания с помощью Интернета, расширенном понимании фактора адресата, включенности научного знания в межкультурную коммуникацию.

Ключевые слова: научный дискурс, адаптивная система, детерминанта, моделирование, процедуры построения знания, аргументация.

Цитирование. Третьякова Т. П. Детерминанты современного научного дискурса в контексте «социализации» знания // Вестник Волгоградского государственного университета. Серия 2, Языкознание. - 2021. T. 20, № 5. - C. 97-107. - DOI: https://doi.org/10.15688/jvolsu2.2021.5.8

\section{Введение}

В современном социуме научная коммуникация существенно меняется. Цифровизация разных сфер общественной жизни, представляющая собой сложное технологическое и социальное явление, приводит к тому, что обмен информацией, в том числе и научной, происходит чрезвычайно быстро и научная информация оказывается доступной широкой аудитории.

Транслируемое за пределами научного сообщества так называемое новое знание становится фактором, объединяющим ученых и всех, кто интересуется научными достижениями (подробно об этом см.: [Болдырев, 2019; Кашкин, 2011; Нефёдов, 2017; Щирова, Гончарова, 2006; Dijk, 2009; Greimas, 1990; Haugh, 2016]), своего рода пиар-ходом для привлечения людей к занятию наукой, способом повышения статуса и престижа научной деятельности, а также средством распространения научной грамотности. В связи с расширением адресатной специализации научного дискурса и его распространением за границы профессионального сообщества (назовем этот процесс «социализацией») появляются даже новые специальности: научный коммуникатор, научный журналист.

Ведущая роль в трансфере научных знаний принадлежит научному тексту. Отметим, что термины «научный текст» и «научный дискурс» применительно к процессу продвижения знаний не дифференцированы в лингвистической литературе, поскольку в настоящее время, благодаря цифровой коммуникации и развитию сетевых технологий, языковые параметры научного текста формируются в динамических интерактивных, то есть дискурсивных, параметрах. Научный текст рассматривается в качестве элемента научной коммуникации, на- правленной на структурирование и передачу знания. Научный текст как структурная единица представления научных достижений традиционно изучался в четко очерченных границах профессиональной коммуникации. В настоящее время такая коммуникация не является замкнутой. Ее открытость обусловлена использованием цифровых каналов обмена информацией через разного рода платформы, а также интенсивной популяризацией знаний в рамках форумов.

В связи с тем, что в силу меняющегося социокультурного контекста язык науки и научный дискурс подвержены трансформациям, имеющим системный характер, меняются и подходы к изучению научного текста. Этот факт становится очевидным, если систематизировать проводившиеся в отечественной лингвистике исследования научного дискурса с точки зрения предмета изучения (направления изучения научного дискурса, например, в конце XX в. см. в таблице).

Отмеченные приоритетные направления изучения научной речи объясняются доминированием методологии структурной и функциональной лингвистики, в рамках которых определяются лексико-грамматические и функционально-стилистические особенности жанров научной речи. Однако, как показано в таблице, проблематика и методики изучения научного дискурса в рамках неформальной интенциональной логики не нашли своего воплощения [Tretyakova, Tretyakov, 1993, p. 467]. Только в конце XX в. в связи с изучением неформальной логики, теории аргументации как разновидности новой риторики, с развитием прагматики и теории дискурса стали появляться исследования дискурсных характеристик языка науки. 
Т.П. Третьякова. Детерминанты современного научного дискурса в контексте «социализации» знания

Основные направления российских исследований языка науки в конце XX в.

Major venues of Russian studies of the language of science in the late $20^{\text {th }}$ century

\begin{tabular}{|c|c|c|c|}
\hline \multirow{2}{*}{$\begin{array}{l}\text { Направление } \\
\text { исследования }\end{array}$} & \multicolumn{2}{|c|}{ Монолог } & \multirow{2}{*}{$\begin{array}{l}\text { Диалог } \\
\text { устный }\end{array}$} \\
\hline & письменный & устный & \\
\hline Стиль / жанр & + & + & + \\
\hline Грамматика / лексика & + & + & + \\
\hline Просодика & - & + & - \\
\hline Прагматика & + & $*$ & * \\
\hline Мето & + & * & - \\
\hline
\end{tabular}

Примечание. Данные приводятся по: [Tretyakova, Tretyakov, 1993]. В таблице использованы следующие обозначения: «+» - активно проходящие исследования; «*»- актуальные только для конца XX в.; «-» - практически полное отсутствие исследований.

Note. Data quoted from: [Tretyakova, Tretyakov, 1993]. The following symbols are used in the table: "+" - current studies; "*”- studies actual for the late $20^{\text {th }}$ century only; "-" - almost no studies.

В начале XXI в. положение изменилось. К отмеченным выше направлениям лингвистического анализа научного текста / дискурса прибавилось изучение отражения в тексте процесса формирования нового знания и его интерпретации.

Цели нашего исследования - определить общие тенденции лингвистического изучения научного дискурса, сложившиеся ко второму десятилетию XXI в. и охарактеризовать дискурс науки с точки зрения производства и представления знания и его включенности в широкую коммуникацию.

\section{Результаты и обсуждение}

\section{Внутренняя и внешняя детерминанты}

Любой ученый, проводящий системный анализ объекта, опирается на понимание системы как сложного единства, в котором могут быть выделены составные части (элементы) и схема связей или отношений между ними (структура). По мнению Г.П. Мельникова, характеристикой любой реальной системы, в том числе и языка, является субстанция, в которую воплощена эта система. «Положение о взаимосвязи структуры и субстанции входит в само определение системы, и так же, как положение о взаимообусловленности статики и динамики, является фундаментом всей концепции» [Мельников, 1972, с. 186]. Данная система относится к адаптивным системам, так как выполняет определенную функцию, которая возникает стихийно, в процессе «естественного отбора», то есть неосознанного предпочтения говорящими тех или иных средств. Исходя из этого, язык науки можно представить как единство текстов (устных и письменных), направленных на производство нового знания. В связи с требованиями четкого моделирования научных текстов, регламентированности устного дискурса и выверенности формулировок адаптивность системы языка науки определена рамками профессиональной коммуникации.

Детерминанта рассматривается как основной параметр системы. Это понятие было применено Г.П. Мельниковым для создания типологической функциональной классификации языков [Мельников, 1972]. Он использовал понятия внешней и внутренней детерминант. Например, рассматривая универсальные свойства разных языков, автор приходит к идее адаптации элементов системы друг к другу, в результате которой создается интенциональный фокус с внутренней детерминантой, трактуемой как специфическое свойство системы, позволяющее настраиваться в том или ином направлении. Внешняя детерминанта - все, что вокруг определяет внутреннюю детерминанту, а внутренняя детерминанта - неизбежность действий внешней детерминанты [Мельников, 1972]. Таким образом, в данной концепции представлено взаимоотношение индивидуального и социального в рамках динамических изменений деятельности человека во времени и пространстве. Все эти процессы так или иначе связаны с процессами воздействия и применением аргументативных технологий, в том числе тех, которые используются в научном дискурсе.

Внутренняя и внешняя детерминанты научного дискурса будут рассмотрены ниже. 


\section{Типология продвижсения знания:}

внутренняя детерминанта

\section{как элемент «социализации" знания}

Внутренняя детерминанта научного дискурса связана с типологией продвижения знания и характеризует когнитивно-лингвистический уровень анализа, отражающий моделирование научного текста с учетом фактора адресата.

Среди подходов, репрезентирующих научный текст как дискурс (см., например: [Славгородская, 1986; Щирова, Гончарова, 2006; Нефёдов, 2016; Чернявская, 2017; Шиян, 2014; Bazerman, 1988; Dijk, 1993; Fairclough, 1992; Viskil, 1994; и др.]), особое внимание уделяется формулировкам убеждения - наиболее важного фактора научной сферы деятельности. Одновременно с этими работами складывалось направление исследования научного дискурса в рамках семиотической теории когнитивного дискурса А. Греймаса [Greimas, Landowski, 1990]. Именно в этой концепции детерминанты научного дискурса проявляются достаточно последовательно как процедуры продвижения знания в группах текстов на трех уровнях (оперативном, инициальном и статусном), представляющих особую иерархию типов текстов, отражающих продвижение знания в направлении «социализации» дискурса.

Первый уровень - оперативный (The Operative Level) - дискурс инициации «создания научного знания» (Production of Knowledge). Независимо от актантов он представляет такую когнитивную деятельность, которая выражается следующими лексическими единицами: noting, observing, examining, clarifying, grouping together, comparing, calculating - или более развернутыми синтаксическими структурами, как например: we see that... it appears that...; if you look... carefully examining [Greimas, Landowski, 1990, p. 47-49]. В некоторых случаях указание на когнитивную деятельность может осуществляться любой таксономической номинацией или сравнительной конструкцией. Если обратиться к системному описанию дискурса, то можно обнаружить, что внутренняя детерминанта представлена в отдельно взятом тексте, направленном на продвижение знания, и реализуется, как показано Е.С. Кубряковой, через коллективную схему уже имеющихся знаний [Кубрякова, 2009, с. 10-11]. Например:

Standpoints need to be brought to the fore and confronted with the doubt and criticism of those concerned to test their acceptability. This means that differences of opinion come into being and are reflected upon and as far as possible discussed by the parties concerned. These differences may be overt and expressed explicitly but they can also remain implicit. However, in order to resolve the differences in a reasonable way, in all cases argumentation is to be advanced to overcome the pertinent doubts and criticisms. Even if a resolution is reached, this resolution is often only temporary and new differences of opinion are bound to arise (p. 1) ${ }^{1}$.

Второй уровень, инициальный, - «уровень открытия» (The Foundational Level) - связан с условиями представления компетентностного знания. Он эксплицируется единицами, отражающими методологию исследования и траектории так называемой операционализации дискурса, то есть направлений поиска потенциального результата исследования: $\partial u c-$ курс открытия (Discourse of Discovery) и дискурс вопросов (Discourse of Questioning), к которому относятся формулировки эпистемологического характера [Greimas, Landowski, 1990, p. 52-54]. Эти типы дискурса связаны с процедурой понимания, начинающегося с интерпретации тех аргументов, которые высказываются по поводу исследования. Большое значение здесь имеют метод и теория. Единицы, указывающие на значимость процедур знания, представляют их как неожиданные события: chance occurrence, conviction, truth, reality, intelligibility, appropriate distance, speculative, dogmatic, accept the standpoint. Дискурс открытия может сопровождаться инференциальными высказываниями:

In argumentative discourse as it occurs in argumentative reality the opening stage corresponds with those parts of the discourse in which the parties involved determine the common starting points on which their exchange will be based and adopt the roles of protagonist and antagonist. Only if some such shared point of departure has been explicitly or implicitly established or silently accepted by the parties does it make sense to undertake an attempt to resolve a difference of opinion by means of argumentation. If there is no point of departure that creates 
possibilities for a constructive exchange of views, having a critical discussion is futile. $<\ldots>$

If no argumentation is advanced and no critical appraisal of this argumentation is given, there is no critical discussion and the difference of opinion will remain unresolved. Because of its crucial role in the resolution process, the argumentation stage is sometimes without any further ado identified with a critical discussion, but for resolving a difference of opinion on the merits the other stages are equally indispensable, so that in argumentation theory these other stages too need to be duly taken into account (p. 37).

Важной характеристикой продвижения знания выступает диалогичность научного дискурса, поскольку она отражает ситуацию принятия решений и понимания. Говорящий становится выразителем эпистемических значений, выразителем исследовательского «я» и носителем знания [Greimas, Landowski, 1990, p. 54]. В этом случае наблюдается связь внутренней детерминанты с внешней. Скрытый учет фактора адресата (стремление быть понятным) является одной из составляющих англоязычного научного стиля.

Дискурс вопросов может служить фактором привлечения внимания и сопровождаться утверждениями:

The qualitative research initially conducted in pragma-dialectics focused primarily on the way in which argumentative moves that are analytically relevant from the perspective of a critical discussion manifest themselves in argumentative discourse. What kind of verbal and other indicators provide a clue as to which of the various kinds of argumentative moves represented in the ideal model of a critical discussion have indeed been made in the discourse? The model of a critical discussion offers a theoretical framework for getting a grip on the analytically relevant aspects of argumentative discourse... (p. 72).

Дальнейшее продвижение знания видится нам, согласно этой теории, как движение к «социализированному» дискурсу, включающему и данные о деятельности первого уровня.

Третий уровень - «уровень проверки: статус знания» (Veridictory Level: The Status of Knowledge). Здесь утверждается существование того предмета, который послужил основой проведения исследования. В текстах этот факт эксплицируется в высказываниях, отражающих когнитивный процесс, то есть выражающих эпистемическую компетенцию: суждения и выводы на основе исследования информации (informative doing) и манипуляций (taxonomic doing), другими словами - создание некоторого дискурса, связанного с объектом исследования. Реализация процедур продвижения знания, таким образом, может рассматриваться как системное описание - вопервых, дистрибутивное или таксономическое, во-вторых, функциональное, трансформационное или генеративное.

Дискурсивность обусловлена модальными высказываниями, направленными на спецификацию некоторых аспектов компетенции когнитивного субъекта, и социальным фактором, связанным с учетом целевого адресата. В этом случае образуется многовекторное взаимодействие, в котором адресат вовлекается в процесс аргументации как «равноправный партнер» [Нефёдов, 2017, с. 601-602] посредством высказываний с инклюзивным местоимением we, модальными словами possibly, perhaps, conceivably или фразами sort of, a little bit, выражающими отсылки к так называемому хеджированию-целенаправленному ослаблению иллокутивной силы высказывания, без которого оно может звучать излишне категорично. Представляется, что здесь реализуется не столько категория вежливости (в понимании В.Е. Чернявской [Чернявская, 2017, с. 93]), сколько умение «обойти острые углы» и избежать категоричности утверждений.

Именно на этом уровне, по мнению А. Греймаса, используется большое количество высказываний, направленных на утверждение экзистенционального характера. К ним относятся следующие:

(a) with a little attention and drawing back - когнитивное высказывание действий (в данном случае - это исключительно информативное высказывание);

(b) we see that... - высказывание состояния, в котором утверждается факт существования объекта (модус «бытия»);

(c) There are political regions just as there are geological regions - за информационной частью следует сравнение, которое необходимо также оценить;

(d) This is true that we instinctively use geographical vocabulary when speaking of 
political parties - оценочное высказывание, завершающее объединенные в единый текст высказывания (a), (b), (c).

Далее А. Греймас проводит анализ дискурса в направлении его типологии по значениям референциального дискурса (this), предикатов существования (there are) к более высокому уровню - так называемой эпистемической модализации (certainty, uncertainty, improbability, probability), высказываниям, выражающим знание (utterance of knowledge), подтверждающим достоверность положений (That is true that...) [Greimas, Landowski, 1990, p. 55-56].

Статусный уровень - проверка представления знаний - зависит от коммуникативной ситуации, которая включает такие структурные компоненты, как отправитель и получатель знаний. Сам научный дискурс строится на определенных логических схемах убеждающего дискурса и в то же время допускает разного рода оценки. Элементы референциального уровня и особенно эпистемической модализации свидетельствуют о модификациях внутренней детерминанты, которая, меняясь под влиянием внешней детерминанты, приобретает все более «разговорный характер». В приведенных примерах эта реализация связана с интерпретацией текста и некоторыми «разночтениями» в этой интерпретации. Как справедливо отметил В.С. Нефедов, «выражая когнитивные целеустановки знания и мнения речевого и познающего субъекта в широком семантическом диапазоне от абсолютной уверенности до сомнения на грани отрицания истинности, эпистемическая модальность и различные ее языковые маркеры выполняют в научных текстах важные дискурсивные функции: они актуализируют взаимодействие конкретного исследователя с соответствующим профессиональным сообществом» [Нефёдов, 2016, с. 144].

В современной когнитивной лингвистике данный фактор связывается с категорией понимания, в том числе индивидуального, и принципиальной диалогичностью процесса логического развертывания высказываний знания и его интерпретации.

По мнению Н.Н. Болдырева, существует два типа языковой интерпретации - первичная и вторичная. Первичная интерпретация, объективная, определяет коллективное знание о мире. Она связана с категоризацией мира. Вторичная интерпретация, субъективная, индивидуальная, обусловлена отношением к пониманию языковых выражений, посредством которых и происходит продвижение знания. Вторичная интерпретация представлена, как полагает исследователь, преимущественно в системе модусных категорий, в частности эвиденциальности, которую автор включает в широкий класс функциональных категорий разных уровней: коммуникативное членение, экспрессивность, тональность, оценочность и др. [Болдырев, 2019, с. 259-260]. Следует также отметить, что в связи с массовым интересом к проблемам искусственного интеллекта особое значение приобретает изучение знакового характера психологической деятельности, в том числе «социализации» научного дискурса. Здесь нельзя не отметить труды Л.С. Выготского (см., например: [Vygotsky, 1980]), которые востребованы в современной науке, так как позволяют определить психологическую составляющую квантования смыслов.

\section{Внешняя детерминанта как элемент} межкультурной научной коммуникации

Внешние условия функционирования научного дискурса связаны с двумя разнонаправленными векторами его развития. Один из них обусловлен жесткими требованиями представления научного материала в публикациях любых форматов, особенно тех, которые используются для получения их автором квалификационного статуса. Более того, глобализационные процессы современного мира, определяющие представление научного знания по строгим канонам, использование англоязычного формата научной статьи, приводят к унификации композиционного и технического оформления научных трудов, которая обусловлена также цифровизацией как способом хранения информации и их интеграцией в общемировую систему знаний, требованиями поисковых систем, что делает необходимым использование разного рода идентификаторов, ключевых слов и аннотаций, в том числе и на английском языке, который в научном общении выполняет функцию метаязыка. Все эти требования фиксируются в инструкциях по 
оформлению научных статей в рецензируемых журналах или студенческих квалификационных работ (см. об этом: [Иваницкая, 2018]). Выбор английского языка оказывается приоритетным для изложения результатов исследований. К внешним факторам изменения научной коммуникации можно отнести и расширенные аннотации на национальном и обязательно на английском языках, особый регламент размещения рецензий на сайтах и много другое. При этом сохраняется культурная специфика научных традиций.

Другой вектор определяется распространением научного знания посредством проектов в формате, например, так называемой ПостНауки (https://postnauka.ru/), направленной на популяризацию фундаментальных научных достижений. Кроме того, используются лекции, курсы, интервью с приглашенными учеными и др. В современном цифровом обществе этот набор форм популяризации науки расширился до проведения разного рода форумов и возобновления работы общества «Знание», учредителем которого является Министерство науки и высшего образования.

Это стало особенно очевидно в прошедший год вынужденной «личной самоизоляции» и перенесения на цифровые платформы семинаров (вебинаров), конференций, школ и симпозиумов. Для научного общения онлайнформат стал не ограничением, а возможностью осуществления диалога. Далее представим те аспекты коммуникации, которые характерны для научного общения в целом и аргументированного продвижения знания как особой устной формы межкультурной коммуникации в частности.

Несмотря на объединяющую функцию научного дискурса, возникают особенности взаимосвязи языка и культуры, которые зависят и от традиций национальных научных школ, и от национальных культур. Этот фактор в некотором смысле может рассматриваться как элемент внешней детерминации при решении научных задач. Очевидным становится действие внешней детерминанты при лингвистическом анализе устной межкультурной научной коммуникации. Так, наблюдаются разные модели поведения при представлении результатов научного исследования. Например, как показали И. Долинина и В. Чеккет- то, культурные различия обнаруживаются между североамериканским и китайским способами начала презентации. Американцы ожидают, что информация о результатах работы будет находиться в самом начале выступления, и, не найдя его там, интерпретируют сообщение как намеренно расплывчатое или крайне непрофессиональное. Представители азиатской культуры, ожидая услышать самую важную информацию лишь в конце сообщения, пропускают существенную часть научной аргументации. В некоторых случаях аргументативность дискурса зависит от доминантной позиции одного из коммуникантов во время общения [Dolinina, Cecchetto, 1997, p. 11-13].

Научная устная коммуникация направлена не на доказательство вводимого знания, а в большей степени на решение таких практических задач, как обоснование предположения, аргументация целесообразности постановки проблемы, выбор между конкурирующими предложениями и т. д. Принципы научного общения включают принципы функционирования коммуникации в целом. К ним относятся постоянные механизмы согласования, приобретающие особую значимость при утверждении платформ коммуникации, регламента проведения научного диалога и пр.

Научная коммуникация достаточно чувствительна к стратегиям соблюдения вежливости: такая коммуникация должна включать общие основания, то есть ситуацию, когда отношения между собеседниками однозначно устанавливаются как отношения равенства (близкого или почтительного), возникающего при существовании определенных научных авторитетов, или превосходства-подчинения, что достаточно редко встречается в современном цифровом контексте общения. Указанные коммуникативные установки касаются не только общения в научной сфере, но и проблем культуры общения в целом [Куссе, Чернявская, 2019; Dijk, 2009; Halliday, 2007; Tretyakova, 2014]. В настоящее время в связи со стремительными изменениями в распространении цифровизации общения, возникшими вследствие пандемии, создаются новые формы эффективного сотрудничества.

В межкультурной коммуникации трудности возникают в трех областях: во-первых, 
принятые стандарты для проецирования упомянутых отношений на конкретные случаи; во-вторых, складывающиеся отношения в каждом конкретном случае; в-третьих, способы кодирования этих отношений. Они соотносимы с принципами социализации, которые предлагает П. Бурдьё, вводя понятие габитуса как системы прочных приобретенных предрасположенностей, диспозиций. Именно диспозиции используются индивидами как исходные установки, которые порождают конкретные социальные практики [Bourdieu, 1991]. Коммуникативные стратегии должны сохраняться в течение всего периода общения, способствуя выравниванию случайных расхождений в проявлениях вежливости и, следовательно, поддерживая межличностный консенсус академического диалога. Нарушение такого консенсуса происходит, например, в онлайнобщении, когда выключается видеокамера и появляется «аватарка».

Сфера применения вежливости как семантической категории достаточно широка. Во многих сферах профессиональной коммуникации, в том числе и научной, прошла грамматикализация единиц, реализующих стереотипы институциональной коммуникации, например обращение друг к другу, выражение одобрения / неодобрения, которые приобретают все более «политически корректный характер» (подробно об этом см.: [Tretyakova, 2014]).

«Социализация» знаний в научной коммуникации, связанная с действием внешней детерминанты, также обусловлена научной тематикой дискурса. Проблема выявления целесообразности включения в дискурс тех или иных тем состоит в том, что необходимо заранее очертить круг критериев их отбора. В некоторых случаях необходимо определить координаты функционально-семантического анализа, к которым та или иная концепция может быть приложима. Это могут быть реальные объекты или нечто необычное, предполагающее определенное конструирование референтов или гипотетических объектов в духе проектов Илона Маска. Выражение отношения строится с применением оценки (положительной или отрицательной), основанной на культурно-ценностных факторах. В частности, появляется «агонистический» стиль общения, то есть стиль, направленный на нега- тивную критику коллег в ущерб конструктивной критике и созданию приемлемых оснований для творческого диалога (см. об этом: [Tannen, 2002]). «Социализация» знания, обусловленная научной тематикой, связана с проблемами создания намеренной отрицательной диспозиции участников научного дискурса, которую также можно отнести к внешней детерминанте, влияющей на потенциал внутренней детерминанты.

\section{Заключение}

Системное представление научного дискурса посредством определения внутренней и внешней детерминант позволило выявить социальную составляющую деятельности исследователей, направленной на продвижение нового знания. Внутренней детерминантой построения дискурса считаются интенциональные конструкции, соотносимые с уровнями представления научного содержания. Вектор их реализации связан с внешней детерминантой, обеспечивающей «социализацию» дискурса, то есть с фактором адресата. Внешняя детерминанта определяется цифровизацией коммуникативного пространства, глобализационными процессами, влияющими на обмен информацией. Субстанция научного дискурса в лингвистическом описании рассматривается как средство «социализации» и получения нового выводного знания с учетом диспозиции автора в контексте взаимного влияния двух детерминант системы.

\section{ПРИМЕЧАНИЕ}

${ }^{1}$ Источником примеров послужила работа Ф. Х. ван Эймерена «Теория аргументации : Прагма-диалектическая перспектива» (Eemeren F. H. van. Argumentation Theory : A Pragma-Dialectical Perspective. Cham : Springer Nature, 2018. 193 p. (Argumentation Library; vol. 33)).

\section{СПИСОК ЛИТЕРАТУРЫ}

Болдырев Н. Н., 2019. Язык и система знаний. Когнитивная теория языка. 2-е изд. М. : Изд. дом ЯСК. $480 \mathrm{c}$.

Иваницкая Е. В., 2018. Статья как элемент научной коммуникации: особенности формирования 
научного авторитета в международном сообществе // Научный редактор и издатель. Т. 3, № 1-2.C. 44-51. DOI: $10.24069 / 2542-0267-2018-$ $1-2-44-51$.

Кашкин В. Б., 2011. Метакоммуникация в пространстве обыденного и научного познания // Вестник Тверского государственного университета. Серия «Филология». № 1. С. 21-27.

Кубрякова Е. С., 2009. В поисках сущности языка // Вопросы когнитивной лингвистики. № 1 (018). C. 5-12.

Мельников Г. П., 1972. Системный подход в лингвистике // Системные исследования. Ежегодник. 1972. М. : Наука. С. 183-204.

Нефёдов С. Т., 2016. Интенциональная структура и модальность научного текста // Филологические науки. Вопросы теории и практики. № 9 (63), ч. 3. С. 141-145.

Нефёдов С. Т., 2017. Рестриктивная аргументация: модальные слова сомнения и общезначимости (на материале немецкоязычных лингвистических статей) // Вестник Санкт-Петербургского университета. Язык и литература. Т. 14, №4. С. 599-610.

Славгородская Л. В., 1986. Научный диалог. Л. : Наука. $167 \mathrm{c}$.

Чернявская В. Е., 2017. Научный дискурс : Выдвижение результата как коммуникативная и языковая проблема. М. : ЛЕНАНД. 144 с.

Шиян Т. А., 2014. К проблеме трансформаций философских и научных дискурсов: модель предметного замыкания // Методология науки и дискурс-анализ / отв. ред. А. П. Огурцов . М. : ИФ РАН. С. 174-204.

Щирова И. А., Гончарова Е. А., 2006. Текст в парадигмах современного гуманитарного знания. СПб. : Кн. дом. 172 с.

Bazerman C., 1988. ShapingWritten Knowledge : TheGenre and Activity of the Experimental Article in Science. [S. 1.] : University of Wisconsin Press. 356 p.

Bourdieu P., 1991. Language and Symbolic Power. Cambridge, Mass. : Harvard univ. press. IX, 302 p.

Dijk T. A. van, 1993. Principles of Critical Discourse Analysis // Discourse and Society. № 4/2. P. 249-283.

Dijk T. A. van, 2008. Discourse and Context: A SocioCognitive Approach. Cambridge : Cambridge University Press. $254 \mathrm{p}$.

Dijk T. A. van, 2009. Society in Discourse. How Context Controls Text and Talk. Cambridge : Cambridge University Press. 287 p.

Dolinina I., Cecchetto, V., 1997. Facework and Rhetorical Strategies in Intercultural Argumentation // OSSA Conference Archive. 23. URL: https:// scholar.uwindsor.ca/cgi/viewcontent. cgi? article $=1870$ \& context $=$ ossaarchive (date of access: 12.09.2020).
Fairclough N., 1992. Discourse and Social Change. Cambridge : Polity Press. 259 p.

Greimas A. J., 1990. The Social Science. A Semiotic View. Minneapolis : University of Minnesota Press. 197 p.

Greimas A. J., Landowski E., 1990. The Pathways of Knowledge// Greimas A. J. The Social Science. A Semiotic View. Minneapolis : University of Minnesota Press. P. 37-58.

Haugh M., 2016. The Role of English As a Scientific Metalanguage for Research in Pragmatics: Reflections on the Metapragmatics of 'Politeness' in Japanese// East Asian Pragmatics. № 1 (1). P. 39-71.

Tannen D., 2002. Agonism in Academic Discourse //Journal of Pragmatics. Vol. 34, iss. 10/11, Oct. Nov. P. 1651-1669. DOI: https://doi.org/10.1016/ S0378-2166(02)00079-6.

Tretyakova T. P., 2014. Discourse Linguistics and Argumentation As Open Systems // Considering Pragma-Dialectics : A Festcshrift for Frans H. van Eemeren on the Occasion of his $60^{\text {th }}$ Birthday. [S. 1.] : Taylor\& Francis. P. 275-286.

Tretyakova T., Tretyakov I., 1993. Russian Studies of the Language of Science Since 1970: A Review Configurations // Journal of Literature, Science and Technology. Vol. 1, № 3. P. 465-473.

Viskil E., 1994. Definition in Argumentative texts // Studies in Pragma-Dialectics / ed. by F. H. van Eemeren, R. Grootendort. Amsterdam : SicSat. P. 79-87.

Vygotsky L. S., 1980. Mind in Society. The Development of Higher Psychological Processes. Cambridge : Harvard University Press. $176 \mathrm{p}$.

\section{REFERENCES}

Boldyrev N.N., 2018. Yazyk i sistema znanya. Kognitivnaya teoriya yazyka [Language and the System of Knowledge. A Cognitive Theory of Language]. Moscow, Izdatelskiy dom YaSK, $480 \mathrm{p}$.

Ivanitskaya E.V., 2018. Statya kak element nauchnoy kommunikatsii: osobennosti formirovaniya nauchnogo avtoriteta $\mathrm{v}$ mezhdunarodnom soobshchestve [Article As the Element of Scientific Communication: Specifics of Formation of Scientific Authority in the International community]. Nauchnyy redaktor $i$ izdatel [Science Editor and Publisher], vol. 3, no. 1-2, pp. 44-51. DOI: https://doi.org/10.24069/25420267-2018-1-2-44-51.

Kashkin V.B., 2011. Metakommunikaciya v prostranstve obydennogo i nauchnogo poznaniya [Metacommunication in Lay and Scientific 
Cognition]. Vestnik Tverskogo gosudarstvennogo universiteta. Seriya «Filologiya». no. 1, pp. 21-27.

Kubryakova E.S., 2009. V poiskakh sushchnosti yazyka [In Search of the Essence of the Language]. Voprosy kognitivnoj lingvistiki [Issues of Cognitive Linguistics], no. 1 (018), pp. 5-12.

Melnikov G.P., 1972. Sistemnyy podkhod v lingvistike [Systemic Approach in Linguistics]. Sistemnye issledovaniya. Ezhegodnik. 1972 [Systemic Language Studies. Yearbook. 1972]. Moscow, Nauka Publ., pp. 183-204.

Nefyodov S. T., 2016. Intencionalnaya struktura i modalnost nauchnogo teksta [Intentional Structure and Modality of a Scientific Text]. Filologicheskie nauki. Voprosy teorii i praktiki. [Philology. Theory \& Practice], no. 9 (63), part 3, pp. 141-145.

Nefedov S.T., 2017. Restrikivnaya argumentatsia: modalnye slova somneneiya i obscheznachimosti (na materiale nemetskoyazychnykh linguisticheskikh statey) [Restrictive Argumentation: Modal Words of Doubt and Shared Knowledge in Academic Linguistic Writing]. Vestnik Sankt-Peterburgskogo universiteta. Yazyk i literatura [Vestnik of SaintPetersburg University. Language and Literature], vol. 14, no. 4, pp. 599-610.

Slavgorodskaya L.V., 1986. Nauchnyy dialog [Scientific Dialogue]. Leningrad, Nauka Publ. 167 p.

Chernyavskaya V.Ye., 2017. Nauchnyy diskurs: Vydvizheniye rezultata kak kommunikativnaya $i$ yazyrovaya problema [Academic Discourse: Promoting the Results As a Communicative and Language Problem]. Moscow, LENAND Publ. 144 p.

Shyian T.A., 2014. K probleme transformatsii filosofskikh i nauchnykh disckursov: model predmentnogo zamykaniya [To the Problem of Transformations in Philosophical and Scientific Discourses: The Model of Subject-Matter Closing].Ogurtsov A.P., ed. Metodologiya nauki $i$ diskurs-analiz. Moscow, IF RAN, pp. 174-204.

Shchirova I.A., Goncharova E.A., 2006. Tekst v paradigmakh sovremennogo gumanitarnogo znaniya [Text in the Paradigms of the Modern Humanities' Knowledge]. Saint Petersburg, Knizhnyy dom Publ. 172 p.

Bazerman C., 1988. Shaping Written Knowledge: The Genre and Activity of the Experimental Article in Science. S. 1., University of Wisconsin Press. 356 p.
Bourdieu P., 1991. Language and Symbolic Power. Cambridge, Mass., Harvard univ. press. IX, 302 p.

Dijk T.A. van, 1993. Principles of Critical DiscourseAnalysis. Discourse and Society, no. 4/2, pp. 249-283.

Dijk T.A. van, 2008. Discourse and Context: A SocioCognitive Approach. Cambridge, Cambridge University Press. 254 p.

Dijk T.A. van, 2009. Society in Discourse. How Context Controls Text and Talk. Cambridge, Cambridge University Press. 287 p.

Dolinina, I., Cecchetto, V., 1997. Facework and Rhetorical Strategies in Intercultural Argumentation. OSSA Conference Archive. 23. URL: https:// scholar.uwindsor.ca/cgi/viewcontent.cgi?article= 1870\&context $=$ ossaarchive (accessed 12 September 2020).

Fairclough N., 1992. Discourse and Social Change. Cambridge, Polity Press. 259 p.

Greimas A.J., 1990. The Social Science. A Semiotic View. Minneapolis, University of Minnesota Press. 197 p.

Greimas A.J., Landowski E., 1990. The Pathways of Knowledge. Greimas A.J. The Social Science. A Semiotic View. Minneapolis, University of Minnesota Press, pp. 37-58.

Haugh M., 2016. The Role of English As a Scientific Metalanguage for Research in Pragmatics: Reflections on the Metapragmatics of 'Politeness' in Japanese. East Asian Pragmatics, no. 1 (1), pp. 39-71.

Tannen D., 2002. Agonism in Academic Discourse. Journal of Pragmatics, vol. 34, iss. 10/11, Oct. Nov., pp. 1651-1669. DOI: https://doi.org/ 10.1016/S0378-2166(02)00079-6.

Tretyakova T.P., 2014. Discourse Linguistics and Argumentation As Open Systems. Considering Pragma-dialectics. A Festcshrift for Frans $H$. van Eemeren on the Occasion of his $60^{\text {th }}$ Birthday. S. 1., Taylor \& Francis, pp. 275-286.

Tretyakova T., Tretyakov I., 1993. Russian Studies of the Language of Science since 1970: A ReviewConfigurations. Journal of Literature, Science and Technology, vol. 1, no. 3, pp. 465-473.

Viskil E., 1994. Definition in Argumentative texts. Eemeren F. H. van, Grootendort R., eds. Studies in Pragma-Dialectics. Amsterdam, SicSat, pp. 79-87.

Vygotsky L.S., 1980. Mind in Society. The Development of Higher Psychological Processes. Cambridge, Harvard University Press. $176 \mathrm{p}$. 
Т.П. Третьякова. Детерминанты современного научного дискурса в контексте «социализации» знания

\section{Information About the Author}

Tatyana P. Tretyakova, Doctor of Sciences (Philology), Professor, Department of English Philology and Translation Studies, St. Petersburg State University, Universitetskaya Emb., 11, 199034 Saint Petersburg, Russia, tretyakova.tp.50@gmail.com, https://orcid.org/0000-0003-2916-8678

\section{Информация об авторе}

Татьяна Петровна Третьякова, доктор филологических наук, профессор кафедры английской филологии и перевода, Санкт-Петербургский государственный университет, Университетская наб., 11, 199034 г. Санкт-Петербург, Россия, tretyakova.tp.50@gmail.com, https://orcid.org/0000-0003-2916-8678 